\title{
Temperature Effect on Read Current in a Two-Bit Nitride-Based Trapping Storage Flash EEPROM Cell
}

\author{
Mu-Yi Liu, Yao-Wen Chang, Nian-Kai Zous, Ichen Yang, Tao-Cheng Lu, Tahui Wang, Member, IEEE, Wenchi Ting, \\ Joseph $\mathrm{Ku}$, and Chih-Yuan Lu, Fellow, IEEE
}

\begin{abstract}
The temperature effect on the read current of a two-bit nitride-storage Flash memory cell is investigated. In contrast to a conventional silicon-oxide-nitride-oxide (SONOS) cell with uniform Fowler-Nordheim (FN) programming, a significant high- $V_{T}$ state read current increase, which results in the read window narrowing at high temperature, is observed in a channel hot electron (CHE) programmed cell. The increment of high- $V_{T}$ state leakage current shows a positive correlation with program/erase threshold voltage window. Since the temperature effect is very sensitive to a locally trapped charge profile, a two-dimensional simulation with a step charge profile is employed to characterize the relationship between current increment and both charge width and charge density.
\end{abstract}

Index Terms-EEPROM, Flash memory, nitride-based trapping storage, temperature effect, trapped charge profile, window narrowing.

\section{INTRODUCTION}

$\mathbf{F}$ LASH memory devices based on the storage of charges in localized trap states in silicon nitride were proposed. By taking the advantages of localized charge trapping in the nitride above source and drain junctions, two-bit storage of a single cell can be achieved by utilizing channel hot electron (CHE) program and band-to-band hot hole erase with a reverse read scheme [1]. Some reliability issues such as cycling endurance and retention have been discussed [2]-[4]. For commercial applications, cell operation at temperature up to $85^{\circ} \mathrm{C}$ is required. The maintenance of sufficient read current window is necessary in a wide range of temperature. In this letter, we will report for the first time on the temperature effect on read current in a trapping storage device. The impact of a trapped charge profile in high-temperature operation will be studied.

Read window narrowing at high temperature is observed and the relationship between high- $V_{T}$ state read current increment at high temperature and the trapped charge profile is discussed. A MXVAND cell [3], [4] with a gate length of $0.36 \mu \mathrm{m}$ and a gate width of $0.2 \mu \mathrm{m}$ is used. The thicknesses of top and bottom oxides are 9 and $5 \mathrm{~nm}$, whereas the interleaving nitride layer is $5.5 \mathrm{~nm}$ thick. Devices are programmed by channel hot electron

Manuscript received February 19, 2004; revised April 16, 2004. The review of this letter was arranged by Editor C.-P. Chang.

M.-Y. Liu, Y.-W. Chang, N.-K. Zous, I. Yang, T.-C. Lu, W. Ting, J. Ku, and C.-Y. Lu are with the Macronix International Ltd., Hsinchu 300, Taiwan, R.O.C. (e-mail: miliu at mxic.com.tw).

T. Wang is with the Department of Electronics Engineering, National Chiao-Tung University, Taipei, Taiwan, R.O.C.

Digital Object Identifier 10.1109/LED.2004.830275

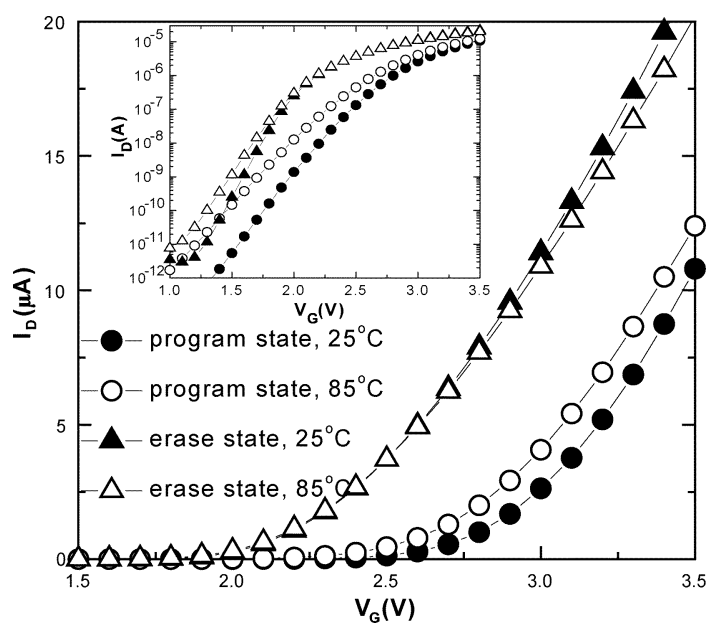

Fig. 1. Measured $I_{D}-V_{G}$ curves for both linear and logarithmic scale, biased at $V_{D}=1.6 \mathrm{~V}$, of two MXVAND cells in erase state and program state at room temperature and high temperature, respectively.

$(\mathrm{CHE})$ injection at $V_{\mathrm{GS}}=11 \mathrm{~V}$. To emulate product operation, the drain bias steps from 3.0 to $4.4 \mathrm{~V}$ with $\Delta V_{D}=0.2 \mathrm{~V}$.

\section{READ CURRENT WINDOW NARROWING}

Fig. 1 shows the $I_{D}-V_{G}$ curves, biased at $V_{D}=1.6 \mathrm{~V}$, of MXVAND cells in erase state and program state at room temperature, and high temperature respectively. Here, the erase state and the program state represent the high bound of low- $V_{t}$ cells and the low bound of high- $V_{T}$ cells in 2-bits per cell product operation. If we read the data of cells at $V_{G}=3 \mathrm{~V}$, the read current window is about $8.77 \mu \mathrm{A}$ at room temperature. But the read current window reduces to $7 \mu \mathrm{A}$ when the cell operates at $85^{\circ} \mathrm{C}$. The degraded read current window may cause an error during data sensing at high temperature especially when the other reliability issues [3], [4] are also taken into account. From the figure, it is found that the window narrowing mainly comes from the read current increment in program state.

When a MOSFET is operated at high temperature, two mechanisms will affect the read current of device. One is the threshold voltage lowering, and the other is the mobility degradation [5], [6]. The former may increase the currents in subthreshold and weakly on regions. The later may degrade the currents in fully on region. As a result, there is usually a crossover between the current-voltage $(I-V)$ curves of device measured at room temperature and high temperature. In Fig. 1, the crossover behavior 


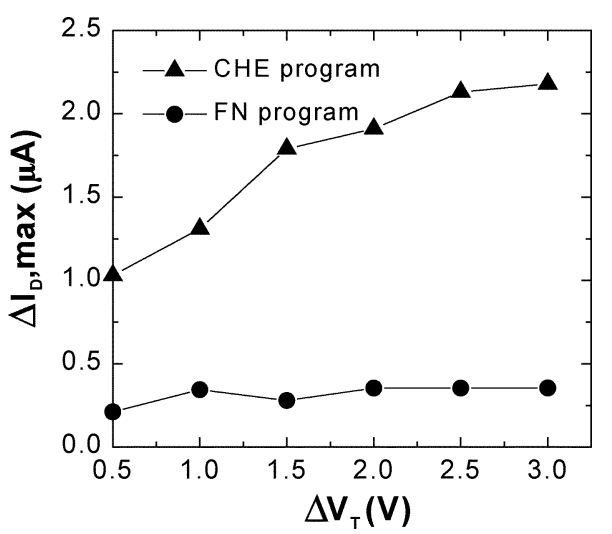

Fig. 2. Temperature effect $\left(\Delta I_{D}\right)$ comparison between CHE programming and FN programming. The CHE programming is at $V_{\mathrm{GS}}=11 \mathrm{~V}, V_{D}$ steps from 3.0 to 4.4 with $\Delta V_{D}=0.2 \mathrm{~V}$, and the $V_{G}$ steps from 17 to $19 \mathrm{~V}$ with $\Delta V_{G}=0.5 \mathrm{~V}$ for FN programming.

of the erase state cell happens at about $V_{G}=2.6 \mathrm{~V}$, and the current increment is insignificant in the weakly on region. But for the cell at program state, which has locally programmed charges at source side, the crossover behavior does not appear even when the applied $V_{G}$ is as high as $3.5 \mathrm{~V}$. Because the impact of our $85^{\circ} \mathrm{C}$ measurement on trapped charge profile is confirmed insignificant (not shown), it is inferred that the localized distribution of programmed charges enhance the subthreshold leakage current and results in a larger shift of $I_{D}-V_{G}$ curve at high temperature. Thus, a delayed crossover point and a larger drain current increment are observed at the program state.

\section{INFLUENCE OF TRAPPED-ChARGE PROFILE}

In Fig. 2, the current increments by using CHE and FN programming are compared. $\Delta I_{D}$ is defined as the maximum read current differencebetweenroomtemperature and $85^{\circ} \mathrm{C}$, and $\Delta V_{T}$ inFig. 2 is the $V_{T}$ window. As programmed $V_{T}$ is increased, the read $V_{G}$ for $\Delta I_{D}$ extraction should also increase to keep the constant gate overdrive. The $\Delta I_{D}$ increases with increasing CHE programmed $V_{T}$ but it keeps almost the same for the cells by FN programming. This result suggests that the temperature effect on read current is enhanced by a localized charge profile and is worsened when the program/erase $V_{T}$ window is increased.

In the subthreshold region, the drain current is dominated by diffusion current [7] and can be expressed as

$$
I_{D}=I_{0} \cdot \exp \left(\frac{q \Psi_{S, \max }}{k T}\right)
$$

where $\Psi s, \max$ is the maximum surface potential. Although the first term $\left(I_{0}\right)$ is a function of temperature, the current increment at high temperature is dominated by the second term due to the exponential dependence. Therefore, the entirely different $\Delta I_{D}$ behavior between FN programming and CHE programming originates from the $\Psi s$, max difference. The characterization of $\Psi s, \max$ in $\mathrm{FN}$ and CHE programmed cells will be discussed in the following.

\section{Relationship BetweEn TRAPPED Charge PROfile AND CURRENT INCREMENT}

To characterize the observation of Fig. 2, a 2-D simulation [8], [9] is utilized to compare the surface potential of cells by

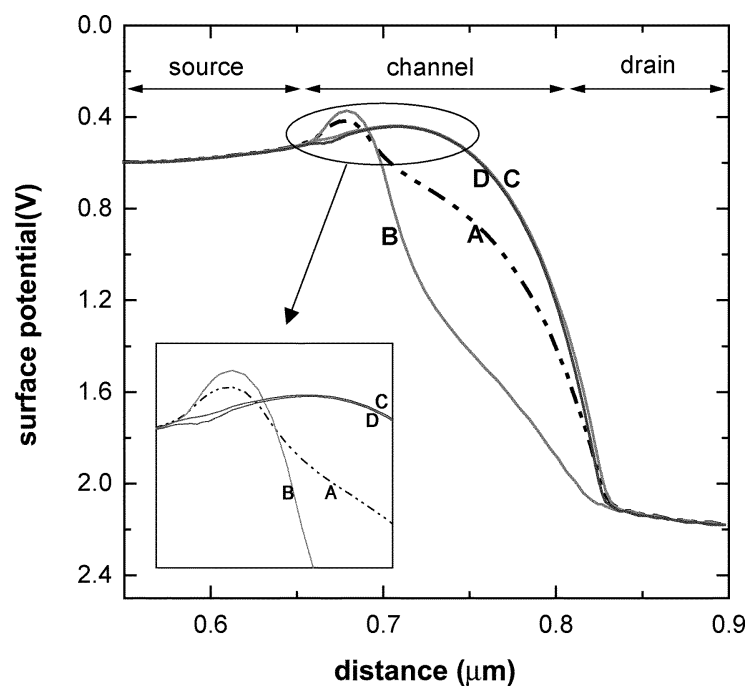

Fig. 3. Simulated surface potential distribution in cells with different $V_{T}$ by FN and CHE programming. (a) CHE, $\Delta V_{T}=0.5 \mathrm{~V}$ at $V_{G}=1.5 \mathrm{~V}$. (b) CHE, $\Delta V_{T}=2.0 \mathrm{~V}$ at $V_{G}=3.0 \mathrm{~V}$. (c) $\mathrm{FN}, \Delta V_{T}=0.5 \mathrm{~V}$ at $V_{G}=1.5 \mathrm{~V}$. (d) FN, $\Delta V_{T}=2.0 \mathrm{~V}$ at $V_{G}=3.0 \mathrm{~V}$. The applied bias is $V_{D}=1.6 \mathrm{~V}$ with a constant gate overdrive.

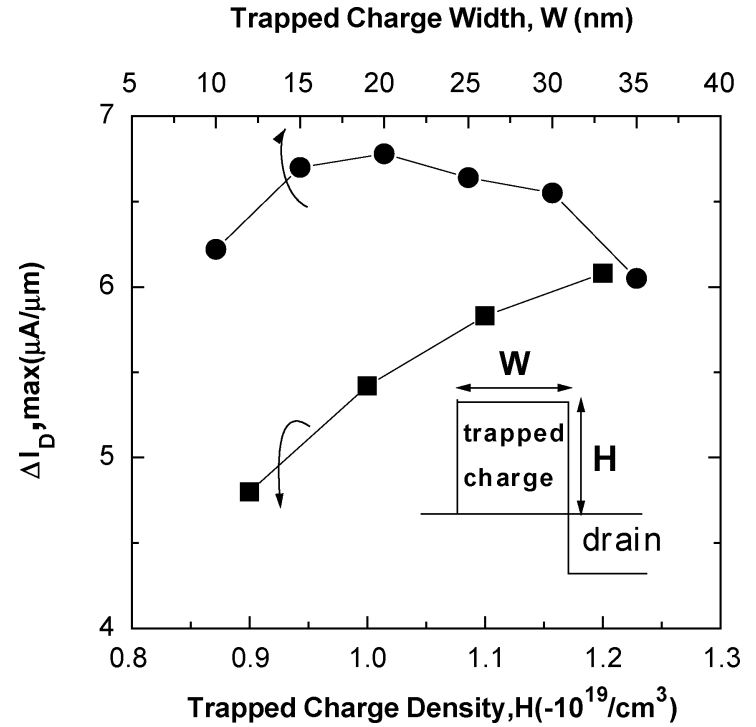

Fig. 4. Relationship between temperature effect $\left(\Delta I_{D}\right)$ and both charge density $(H)$ and charge width $(W)$. The charge density is modified with a fixed width $30 \mathrm{~nm}$ over channel, and the charge width is modified with a fixed density $-2 * 10^{19}\left(1 / \mathrm{cm}^{3}\right)$ over channel.

CHE programming and FN programming. Fig. 3 shows the simulated surface potential distribution of cells with different $V_{T}$ by FN and CHE programming. One can see that the cells by FN programming have a constant $\Psi s$, max due to the constant gate overdrive. As a result, similar $\Delta I_{D}$ is obtained in the FN programmed cells. As to the CHE programmed cells, the $\Psi s$, max are dominated by the local potential barrier, which is pulled high by the local trapped charge. Consequently, if the same $\Delta V_{T}$ are programmed by FN injection and CHE injection, the CHE programmed cell would have a larger $\Psi s$, max and much more serious temperature effect. In addition, $\Psi s, \max$ is increased with increasing CHE programmed $V_{T}$ even the constant gate overdrive is adopted, as shown in Fig. 3. Thus, $\Delta I_{D}$ is increased with increasing CHE programmed $V_{T}$. 
To further clarify how the temperature effect is influenced by a localized charge profile, the relationship between $\Delta I_{D}$ and both the charge density and the charge width are characterized by simulation. As illustrated in Fig. 4, when the charge density is increased with a fixed width, the $\Delta I_{D}$ is increased due to the increased $\Psi s, \max$. In addition, when the charge distribution is narrowed with a fixed density, the programmed $V_{T}$ becomes smaller and only a smaller $V_{G}$ should be applied to extract $\Delta I_{D}$. It leads to a larger $\Psi s, \max$ and the increased $\Delta I_{D}$. As the charge width is narrower than a certain value, $\Psi s$, max would reduce substantially due to drain-induced barrier lowering. Thus, the $\Delta I_{D}$ turns to decrease when the charge distribution is narrower than a certain width.

\section{CONCLUSION}

In this work, the temperature effect of a two-bit nitride-storage Flash memory cell is investigated. A significant program-state read current increment in a CHE programmed cell is observed at high temperature. It degrades the read current window and impacts on data sensing. A localized charge profile that enhances the subthreshold leakage at high temperature may be the root cause of this current increment. From two-dimensional simulation results, the current increment becomes larger as the charge density is higher or the charge distribution is narrower in a certain range.

\section{REFERENCES}

[1] B. Eitan, P. Pavan, I. Bloom, E. Aloni, A. Frommer, and D. Finzi, "NROM: A novel localized trapping, 2-bit nonvolatile memory cell," IEEE Electron Device Lett., vol. 21, pp. 543-545, Nov. 2000.

[2] M. K. Cho and D. M. Kim, "High performance SONOS memory cells free of drain turn-on and over-erase: compatibility issue with current Flash technology," IEEE Electron Device Lett., vol. 21, pp. 399-401, Aug. 2000.

[3] W. J. Tsai, N. K. Zous, C. J. Liu, C. C. Liu, C. H. Chen, T. H. Wang, S. Pan, C. Y. Lu, and S. H. Gu, "Data retention behavior of a SONOS type two-bit storage Flash memory cell," in IEDM Tech. Dig., pp. 32.6.1-32.6.4

[4] C. C. Yeh, W. J. Tsai, T. C. Lu, S. K. Cho, T. Wang, S. Pan, and C.-Y. $\mathrm{Lu}$, "A modified read scheme to improve read disturb and second bit effect in a scaled MXVAND Flash memory cell," in Proc. Non-Volatile Semiconductor Memory Workshop, 2003, pp. 44-45.

[5] F. S. Shoucair, "Design considerations in high temperature analog CMOS integrated circuits," IEEE Trans. Comp., Hybrids, Manufact. Technol., vol. CHMT-9, pp. 242-251, Sept. 1986.

[6] - "Scaling, subthreshold, and leakage current matching characteristics in high-temperature $\left(25^{\circ} \mathrm{C}-250^{\circ} \mathrm{C}\right)$ VLSI CMOS devices," IEEE Trans. Comp., Hybrids, Manuf. Technol., vol. 12, pp. 780-788, Dec. 1989.

[7] T. A. Fjeldly, "Threshold voltage modeling and the subthreshold regime of operation of short-channel MOSFET's," IEEE Trans. Electron Device, vol. 40, pp. 137-145, Jan. 1993.

[8] E. Lusky, Y. Shacham-Diamand, I. Bloom, and B. Eitan, "Characterization of channel hot electron injection by the subthreshold slope of NROM device," IEEE Electron Device Lett., vol. 22, pp. 556-558, Nov. 2001.

[9] E. Lusky, I. Bloom, and B. Eitan, "Investigation of the spatial distribution of CHE injection utilizing the subthreshold slope and the Gate induced drain leakage (GIDL) characteristics of the NROM device," in Proc. Non-Volatile Semiconductor Memory Workshop, 2003, pp. 48-49. 